\title{
Spontaneous cerebrospinal fluid rhinorrhea in patient with Pallister-Hall syndrome
}

\author{
Milicevic Mihajlo ${ }^{1,2}$, Aleksic Vuk ${ }^{1}$, Ilic Rosanda ${ }^{1,2}$ \\ Scepanovic Vuk ${ }^{1}$, Stanimirovic Aleksandar ${ }^{1}$, \\ Paunovic Aleksandra ${ }^{1}$, Milic Marina, Nedeljkovic \\ Zarko, Matteo Sacco ${ }^{1}$, Grujicic Danica ${ }^{1,2}$ \\ 1 Neurosurgery Clinic, Clinical Center of Serbia, Belgrade, Serbia, \\ SERBIA \\ 2 Faculty of Medicine, University of Belgrade, Belgrade, SERBIA
}

\begin{abstract}
Pallister-Hall syndrome (PHS) is extremely rare autosomal dominant disorder with typical clinical features such as presence of polydactyly, hypothalamic hamartoma, bifid epiglottis, anal, renal, genitourinary and pulmonary abnormalities, mainly located in the midline of the body. Spontaneous cerebrospinal fluid (CSF) rhinorrhea is also a rare condition, and it is used to describe nasal discharge of CSF unrelated to previous trauma, localized tumour, surgery, or previous radiation therapy. The exact cause is not yet fully understood.

We report a case of adult female patient previously diagnosed with PHS and late onset of spontaneous nasal liquorrhea, due to defect in the anterior skull base. Although it can be incidental finding, we believe that described defect in the body midline can be another presentation of congenital craniofacial abnormality which are common in PHS.
\end{abstract}

\section{INTRODUCTION}

Pallister Hall syndrome (PHS) is extremely rare autosomal dominant disorder usually diagnosed in infants and children, but it can be seldomly seen in adulthood (1). In 1980, Judith Hall and Philip Pallister described 6 cases of infants with a neonatally lethal malformation syndrome of hypothalamic hamartoblastoma, postaxial polydactyly, and imperforate anus, and after several simmilar cases were described by other authors, this syndrome was named after Pallister and Hall $(2$, 3). Typical clinical features of PHS are presence of polydactyly and hypothalamic hamartoma, however, in recent years, many patients were reported expanding the phenotype, and more rare features may also be seen, such as bifid epiglottis, anal, renal, genitourinary, and pulmonary abnormalities, nonpolydactyly skeletal anomalies, and developmental delay. Even rearly epilepsy, hypopituitarism, aganglonosis of colon, congenital cardiac defect, and adrenal abnormalities can also be found (4). Clinical suspicion of PHS is confirmed by genetic testing of the GLI3 zinc finger transcription factor

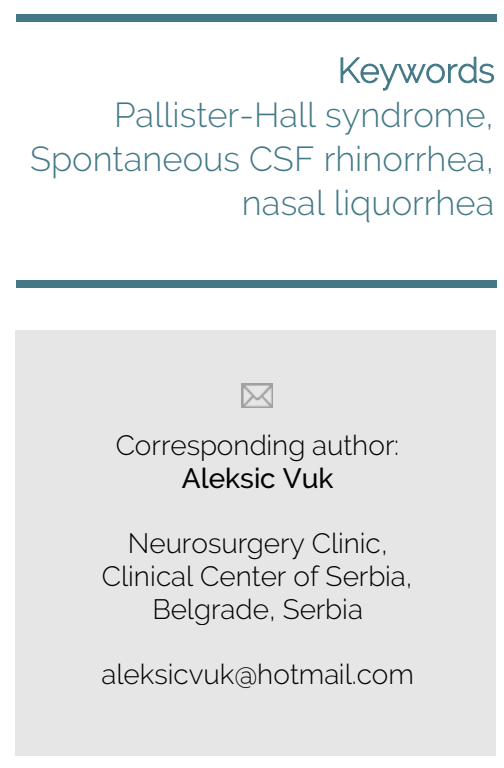

Copyright and usage. This is an Open Access article, distributed under the terms of the Creative Commons Attribution Non-Commercial No Derivatives License (https://creativecommons org/licenses/by-nc-nd/4.0/) which permits noncommercial re-use, distribution, and reproduction in any medium, provided the original work is

unaltered and is properly cited.
The written permission of the Romanian Society of Neurosurgery must be obtained for commercial re-use or in order to create a derivative work.

ISSN online 2344-4959

(C) Romanian Society of Neurosurgery

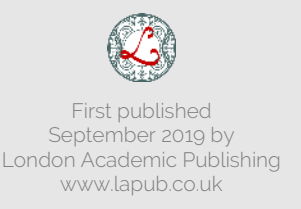


gene on $7 p 14$ (2). The importance of this gene located on chromosome 7 is that it regulates downstream genes of the hedgehog pathway important to the formation of the neural tube, otic vesicles, craniofacial structures, and distal limb buds (4).

We report a case of adult female patient previously diagnosed with PHS and late onset of spontaneous nasal liquorrhea.

\section{CASE REPORT}

We present a case of a 56-year-old woman admitted to the Neurosurgery Clinic of the Clinical Center of Serbia, with signs of spontaneous nasal liquorrhea. She was born from healthy and non-consanguineous parents after normal pregnancy and delivery. On birth examination, she was found to have polysyndactyly on both hands. At the age of 2, she was operated, and ablation of redundant fingers and soft tissue was performed with surgical reconstruction and good appearance in size and shape of both hands was achieved (Picture 1). After few years, in early childhood, patient developed epilepsy with laughing, gelastic seizures, and she was placed on two antiepileptic drugs after which seizures resolved. Later on, during fertility testing she was found to have bilateral renal hypoplasia, as well as uterine hypoplasia. In the late 40 's, during the clinical testing due to signs of polyneuropathy, suspicion of PHS was made by attending neurologist, and brain magnetic resonance imaging (MRI) showed typical hypothalamic hamartoma (Picture 2). Patient underwent genetic testing, and mutation of GLI3 gene in the short arm of chromosome 7 was found.

At the age of 50, the patient noticed spontaneous watery discharge from left nostril, and occasional sweet taste in her mouth, usually in the morning or after a long bed rest. There was no history of head trauma. After 4 years of neglecting this symptom patient was referred to our hospital. At admission her neurologic exam was indicative of distal polineuropathy without signs of motor weakness. High glucose concentration, and detection of $\beta$-2transferrin in clear fluid collected from left nostril confirmed the diagnosis of cerebrospinal fluid rhinorrhea. Head MRI was performed and communication of the frontobasal liquor space with the upper nasal corridor at the level of the left olfactory nerve was found. Also, presence of hypothalamic hamartoma in the projection of tuber cinereum, $19 \times 13 \mathrm{~mm}$ in diameter was confirmed. A non-contrast $C T$ scan with bone window revealed mucosal thickening in the left cribriform plate in conjunction with the olfactory fila presenting possible defect in the adjacent skull base (Picture 3). We performed endonasal endoscopic repair of CSF rhinorrhoea under general anaesthesia. Operatively identified bone defect in the left cribriform plate with signs of CSF leak was closed by dura matter, fascia lata, and tissue glue. The mucosa of middle turbinate and adjacent septum was made raw and bolgerisation was performed. Valsalva manoeuvre did not show signs of CSF leakage. After haemostasis was achieved nose was bagged for five days. Avoidance of coughing or sneezing, the use of stool softeners, elevation of the head, and bed-rest were given in the post-operative period. Patient was on prophylactic antibiotics for one week. On the 5th post-operative day patient was discharged from the hospital. At 2 year follow-up patient is asymptomatic, without signs of nasal liquorrhea. Also, nasal cavities healed well, with no breathing difficulties.

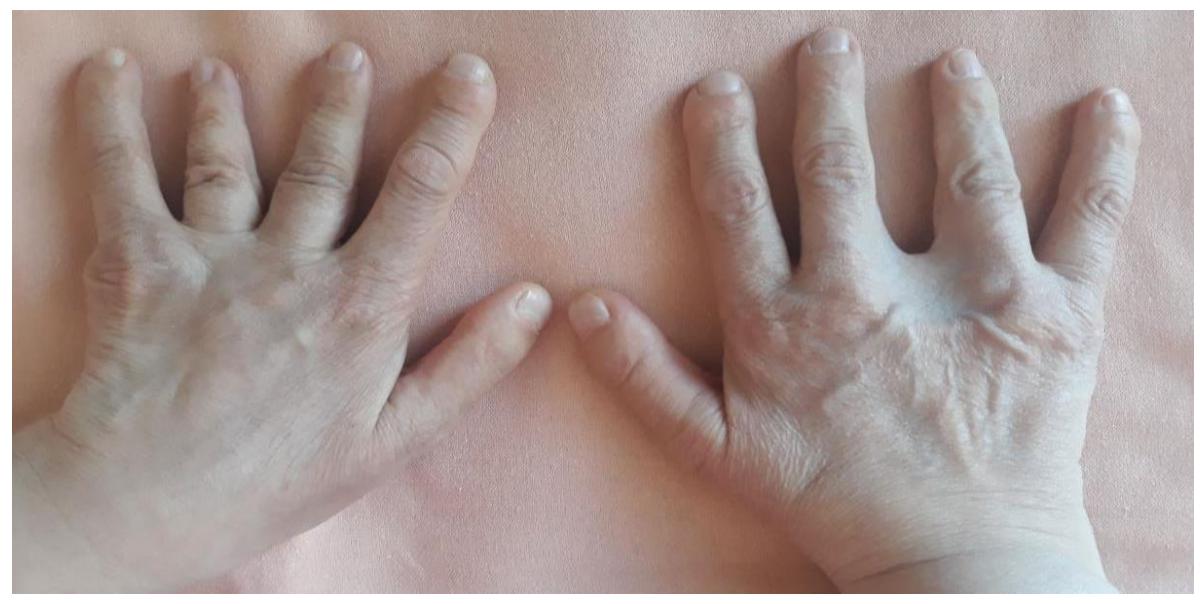

Picture 1. Present look of patient's hands, 54 years, after ablation of redundant fingers and soft tissue due to polysyndactyly. 


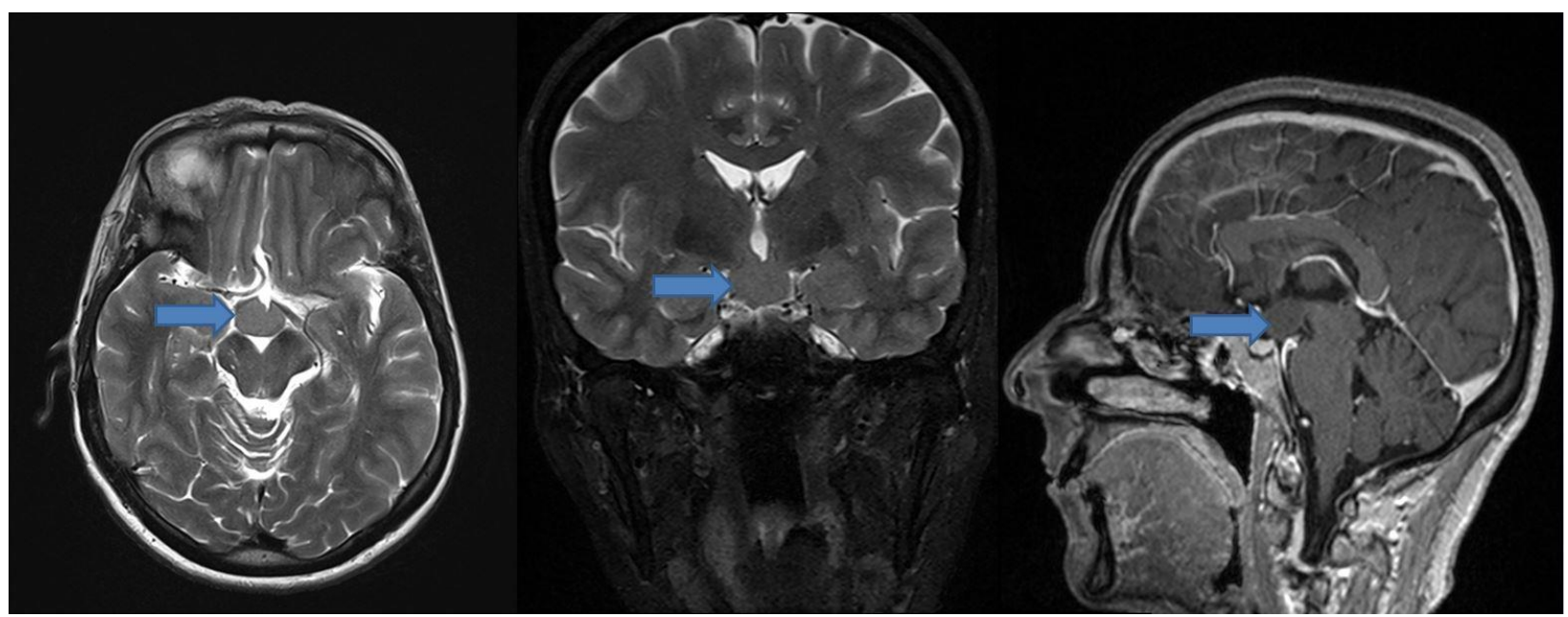

PICTURE 2. Brain MRI showing hypothalamic hamartoma in our patient

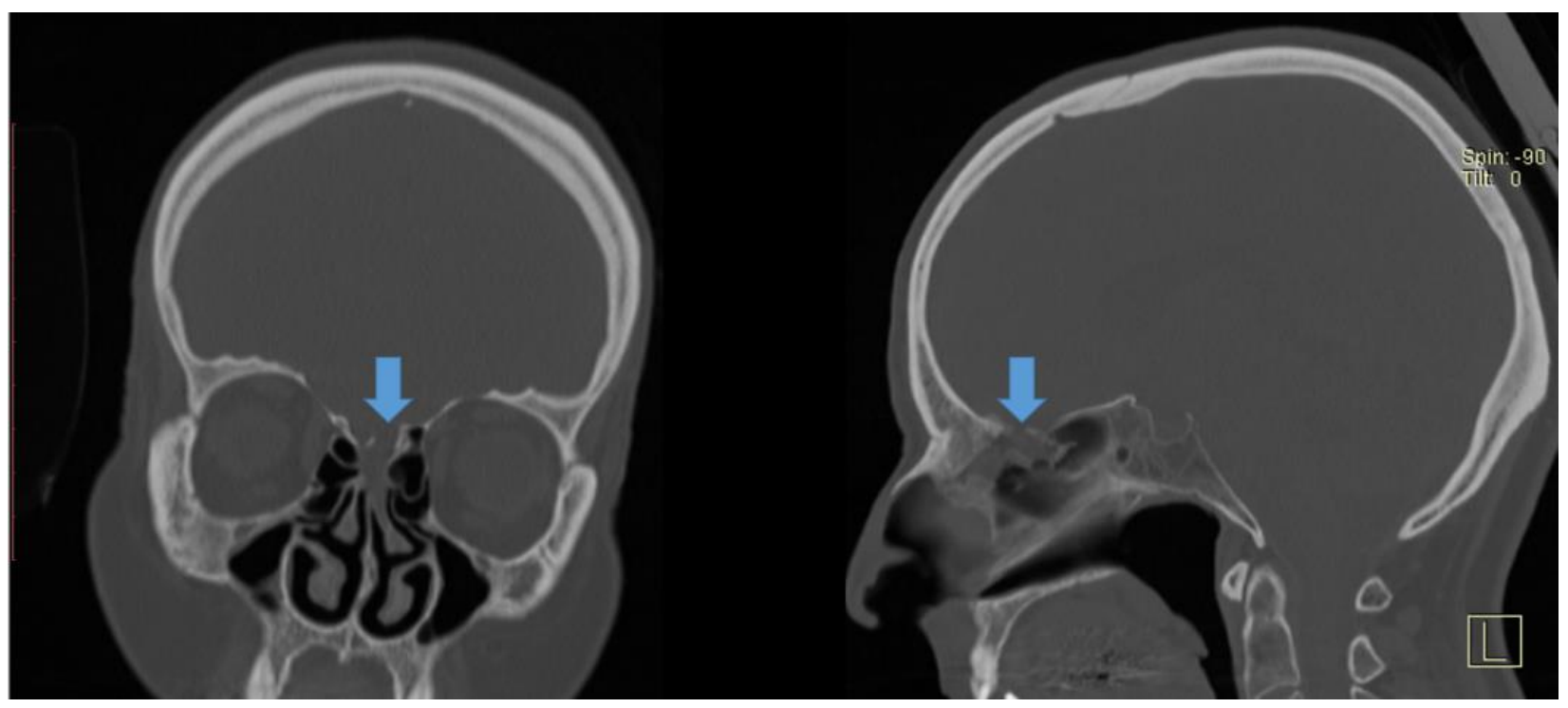

PICtURE 3. CT scan (bone window) showing mucosal thickening in the left cribriform plate in conjunction with the olfactory fila presenting defect in the adjacent skull base

\section{DISCUSSION}

PHS is extremely rare autosomal dominant disorder usually diagnosed in infants and children, but it can be seldomly seen in adulthood (1). Dramatic beginning of PHS started in 1980, when Judith Hall and Philip Pallister described 6 lethal cases of infants with typical combination of hypothalamic hamartoblastoma, postaxial polydactyly, and imperforate anus $(2,3)$. Today, this clinical features of PHS in the terms of the clinical findings are considered to be just a tip of the iceberg, since many others rare features may also be seen, such as bifid epiglotis, polydactyly, dysmorphic face in the form of small ears pointing backwards, small retroverted nose, flat nasal bridge, and small tongue. Also, different cardiac, anal, renal, adrenal, genitourinary, and pulmonary abnormalities can be found. Hypothalamic hamartomas are typically found, and can be asymptomatic or in the origin of epilepsy, hypopituitarism, lethargy, hypoglycaemia, electrolyte dysfunction, and metabolic acidosis. So far, about 100 cases of PHS with variable manifestations are reported in literature (4-6). We reported a case of adult female patient previously diagnosed with PHS and late onset of spontaneous nasal CSF rhinorrhoea. To our knowledge this is a 
first case of PHS patient with skull base bone defect, presented with spontaneous cerebrospinal fluid rhinorrhoea. Also, this is the first described case of PHS in Serbian population according to our knowledge. Our patient is a 56-year-old woman with previously operated polysyndactyly on both hands, well controlled epilepsy with typical gelastic seizures, bilateral renal and uterine hypoplasia, and hypothalamic hamartoma. These are usual signs and symptoms found in PHS. About ninety-five percent of PHS patients have GLI3 gene mutation in the short arm of chromosome 7 (6). This was also case in our patient, since definite diagnosis was confirmed after molecular testing showing mutation of GLI3 gene.

Hypothalamic hamartoma represents a benign tumour of the hypothalamus and does not need treatment from a tumour biological perspective. However, other problems can be associated with hypothalamic hamartoma such as pharmacoresistant epilepsy, behavioural problems, and endocrine disturbances (7). An MRI of the brain in our patient revealed presence of hypothalamic hamartoma in the projection of tuber cinereum, $19 x$ $13 \mathrm{~mm}$ in diameter. In presented case patient developed epilepsy with laughing, gelastic seizures. After introduction of two different antiepileptic drugs, our patient was in total remission.

The term spontaneous CSF rhinorrhoea has been used to describe nasal discharge of CSF unrelated to previous trauma, localized tumour, surgery, or previous radiation therapy, and it represents a rare medical condition (8). Our patient had first onset of watery discharge from left nostril at the age of 50, without history of previous trauma, and after neglecting symptoms for about 4 years, patients was referred to our hospital. Also, other possible causes of spontaneous CSF rhinorrhoea were excluded in our patient.

In the study of Schuknecht et al, more than 70\% of patients with spontaneous CSF leak had osteodural interruption at the site of cribriform plate leading into the olfactory cleft (8). This was also a case in our patient.

In the case of osteodural interruption surgical repair is recommended to prevent complications such as meningitis. Endonasal endoscopy is the preferred and minimally invasive approach and it has been accepted worldwide as the method of choice. The advantages of this approach are: excellent visualization, precise placement of graft, minimal surrounding tissue damage, preservation of olfactory nerves and their function, shortened operating time, and faster recovery. This approach has a high success rate up to $97 \%(9,10)$. We performed surgical endonasal endoscopic repair of previously found osteodural deffect along the cribriform plate leading into the left olfactory cleft. On two years fallow-up, patient is without signs of CFS rhinorrhoea recurrence.

PHS is a rare, autosomal dominant, genetic disorder with a mutation in the GLI3 gene on the short arm of chromosome 7. This gene regulates pathways important for the formation of the neural tube, craniofacial structures, otic vesicles, and limbs (11). Many different congenital abnormalities are found in patients with PHS, and hypothalamic hamartoblastoma, bifid epiglottis, polydactyly, epilepsy, imperforate anus and other genital anomalies being most common, with predominance of anomalies in the midline of the body $(4,12)$. About 100 case reports of PHS are found in the literature, but to our knowledge this is the first case of PHS with spontaneous CSF rhinorrhoea, due to defect in the anterior skull base. Although it can be incidental finding, we believe that described defect in the body midline can be another presentation of congenital craniofacial abnormality which are common in PHS. Endoscopic endonasal approach is minimally invasive and successful treatment approach for described congenital osteodural defect.

\section{REFERENCES}

1. Talsania M, Sharma R, Sughrue ME, Scofield RH, Lim J. Familial Pallister-Hall in adulthood. Neuro Endocrinol Lett. 2017;38(5):329-331.

2. Courtney E, Swee DS, Ishak D, Ngeow J. A delayed diagnosis of Pallister-Hall syndrome in an adult male following the incidental detection of a hypothalamic hamartoma. Hum Genome Var. 2018;5:31.

3. Hall JG, Pallister PD, Clarren SK, Beckwith JB, Wiglesworth FW, Fraser FC, Cho S, Benke PJ, Reed SD. Congenital hypothalamic hamartoblastoma, hypopituitarism, imperforate anus and postaxial polydactyly--a new syndrome? Part I: clinical, causal, and pathogenetic considerations. Am J Med Genet. 1980;7(1):47-74.

4. Hayek F. Pallister-Hall syndrome with orofacial narrowing and tethered cord: a case report. J Med Case Rep. 2018;12(1):354.

5. Biesecker LG, Graham JM Jr. Pallister-Hall syndrome. J Med Genet. 1996;33(7):585-9. 
6. Chandra SR, Daryappa MM, Mukheem Mudabbir MA, Pooja M, Arivazhagan A. Pallister-Hall Syndrome. J Pediatr Neurosci. 2017;12(3):276-279.

7. Mundlos S, Horn D, Mundlos S, Horn D. Limb Malformations. Berlin: Springer; 2014. Pallister-Hall Syndrome; pp. 61-62.

8. Schuknecht B, Simmen D, Briner HR, Holzmann D. Nontraumatic skull base defects with spontaneous CSF rhinorrhea and arachnoid herniation: imaging findings and correlation with endoscopic sinus surgery in 27 patients. AJNR Am J Neuroradiol. 2008;29(3):542-9.
9. Kljajić V, Vuleković $P$, Vlaški L, Savović S, Dragičević D, Papić V. Endoscopic repair of cerebrospinal fluid rhinorrhea. Braz J Otorhinolaryngol. 2017;83(4):388-393.

10. Gonen L, Monteiro E, Klironomos G, Alghonaim Y, Vescan A, Zadeh G, Gentili F. Endoscopic Endonasal Repair of Spontaneous and Traumatic Cerebrospinal Fluid Rhinorrhea: A Review and Local Experience. Neurosurg Clin N Am. 2015;26(3):333-48.

11. Hall JG. The early history of Pallister-Hall syndromeBuried treasure of a sort. Gene. 2016;589(2):100-103.

12. Biesecker LG, Graham JM, Jr. Pallister-Hall syndrome. J Med Genet. 1996;33(7):585. 